\title{
Learning in a Smart City Environment
}

\author{
R. Nikolov ${ }^{1}$, E.Shoikova ${ }^{1}$, M. Krumova ${ }^{2}$, E. Kovatcheva ${ }^{1}$, V. Dimitrov ${ }^{1}$ and A.Shikalanov ${ }^{1}$ \\ 1. State University of Library Studies and Information Technologies \\ 2. Technical University - Sofia
}

\begin{abstract}
Advances in technology in recent years have changed the learning behaviors of learners and reshaped teaching methods and learning environments. The purpose of this paper is to overview a foundational framework and provide models for the planning and implementation of smart learning environments. Introduction is focused on analysis of emerging industries and new types of jobs that are requiring future personnel to be well equipped to meet the need of the expansion requirements of these industries and keep up with their development needs. Gartner's 2015 Hype Cycle for Emerging Technologies identifies the computing innovations such as Internet of Things, Advanced Analytics, Machine Learning, Wearables etc. that organizations should monitor. Learners and students, being the future drivers of these industries, are the main human resource to fulfill the vacancies of these work forces. Constant improvements in and re-evaluation of the curriculum taught to the learners have to be done regularly to keep the learners up-to-date in fulfilling the requirements of these industries and corporations. Universities benefit from these thinking "out of the box practices" by equipping students with work force experience that involves more hands on task with real life infrastructures. Section 2 looks at future Internet domain landscape that comprises a great diversity of technology related topics involved in the implementation of Smart Learning Environments. The purpose of section 3 is to overview a foundational framework and major considerations for the planning and implementation of smart learning environments, behind which is the convergence of advances and developments in social constructivism, psychology, and technology. Section 4 introduces the smart learning models, which are developed to reflect the dynamic knowledge conversion processes in technology enabled smart learning environments. The last section presents a case study of a learning scenario entitled "Monitoring the environmental parameters in a Smart City" as an illustration of experimental learning on Internet of Things, which proofs the power of FORGE (forging online education through FIRE) FP7 project methodology and infrastructure for building remote labs and delivering them to students.
\end{abstract}

Key words: Smart city, smart learning environment, full context awareness, big data and learning analytics, autonomous decision-making, SECI, learning scenario, forging online education through FIRE.

\section{Introduction}

New forms of industries and new types of jobs are emerging, requiring future personnel to be well equipped to meet the need of the expansion requirements of these industries and keep up with their development needs. Gartner's 2015 Hype Cycle for Emerging Technologies identifies the computing innovations that organizations should monitor (see Fig. 1). Learners and students, being the future drivers of these industries, are the main human resource to fulfil the vacancies of these work forces. Constant improvements in and re-evaluation of the curriculum taught to the learners have to be done

Corresponding author: Roumen Nikolov, professor, Ph.D., research fields: computer science, software engineering, internet of things, big data, smart city, e-learning. regularly to keep the learners up-to-date in fulfilling the requirements of these industries and corporations. Universities benefit from these thinking "out of the box practices" by equipping students with work force experience that involves more hands on task with real life infrastructures.

Today, as education systems are currently undergoing significant change brought about by emerging reform in pedagogy and technology, our efforts have sought to close the gap between technologies as educational additive to effective integration as a means to promote and cultivate student centred, inquiry based and project based learning. Moving forward, many of the advances in education will be brought about by further integration of personalised learning into the smart learning environment, 


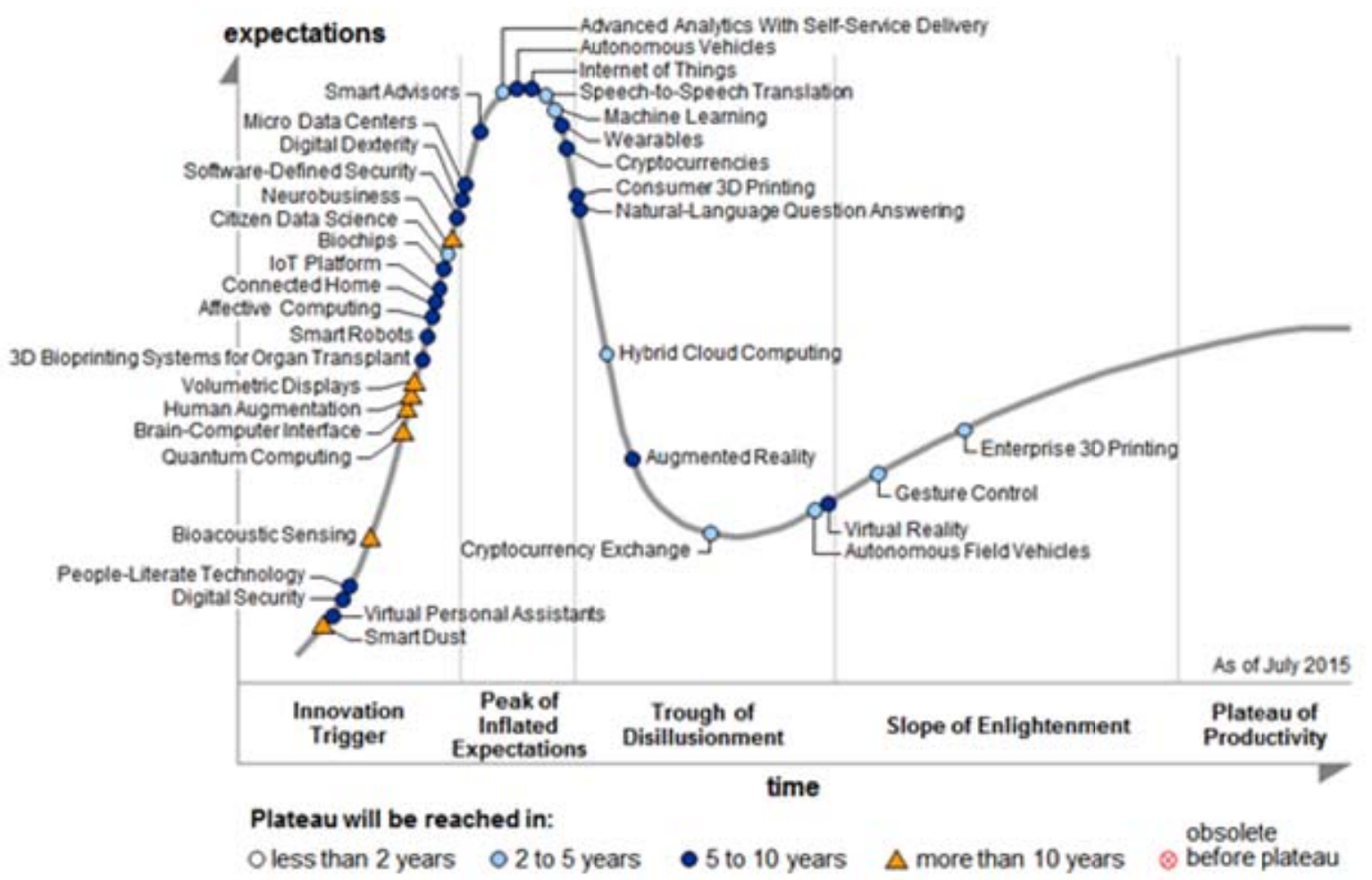

Fig. 1 Gartner's 2015 hype cycle for emerging technologies.

such as ubiquitous access to technology through continuously shifting mobile devices and mobile platforms, cloud based services, big data, and dispersed learning environments will further emphasise the affordances of learning technologies. These changes are also being impacted by broader trends including population shifts, economics, employment, and other societal shifts.

\section{Smart City Concept}

This section covers Smart City definitions as well as some of those technology trends that are most connected to the development of Smart Cities and Smart Learning Environments.

\subsection{Definitions}

There are many definitions for Smart Cities in use globally[1].Smart City is a new concept and a new model, which applies the new generation of information technologies, such as the internet of things, cloud computing, big data and geospatial data integration, to facilitate the planning, construction, and management of smart services. Developing Smart Cities can benefit synchronised development, industrialisation, informationisation, urbanisation and agricultural modernisation and sustainability of cities development. Smart City is a term denoting the effective integration of physical, digital and human systems in the built environment to deliver a sustainable, prosperous and inclusive future for its citizens. The "smartness" of a city describes its ability to bring together all its resources, to effectively and seamlessly achieve the goals and fulfil the purposes it has set itself. A smart city can be viewed as a combination of four Internets or networks: Internet of Things, Internet of People, Internet of Data and Internet of Services. The emphasis is put on the system integration and synergistic characteristic of a smart city (Fig. 2). Such a view illustrates succinctly the "glue”, or the system integration property that ICT provides in smart cities.

An enterprise architecture view emphasises from a domain and outcome perspective, and presents how the ICT in a Smart City would break value by breaking 


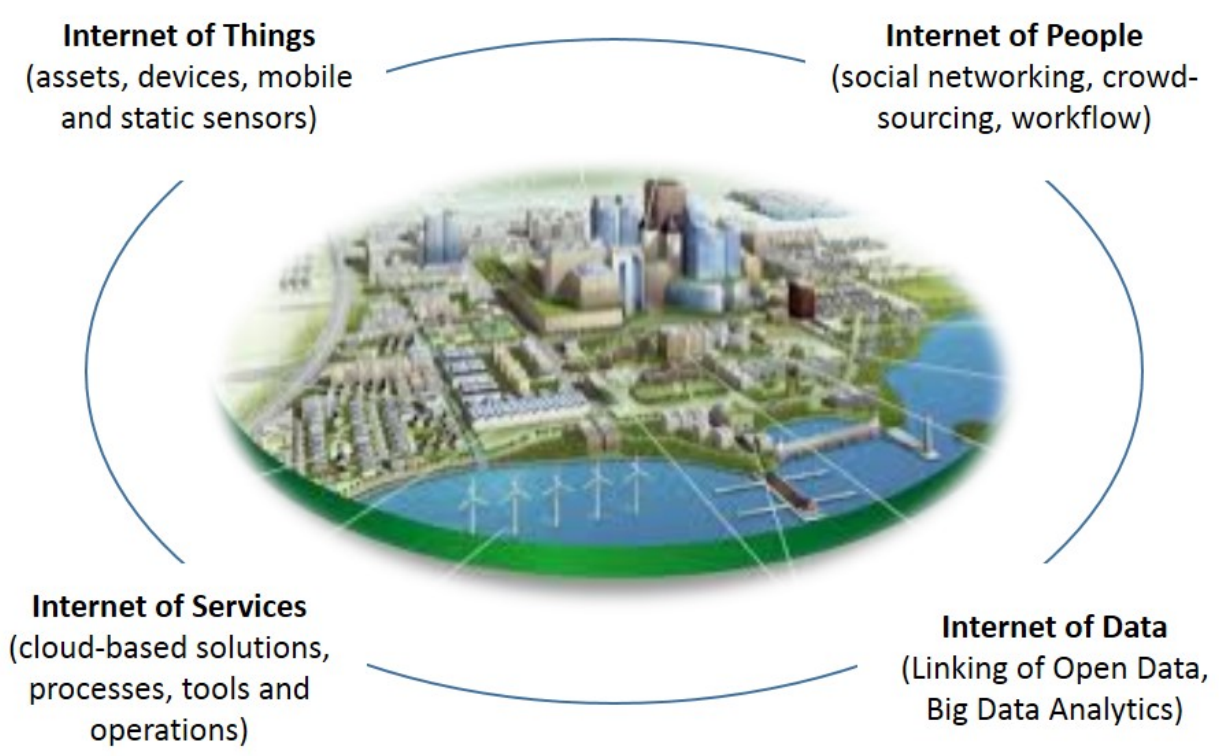

Fig. 2 The smart city as a set of "Internets" .

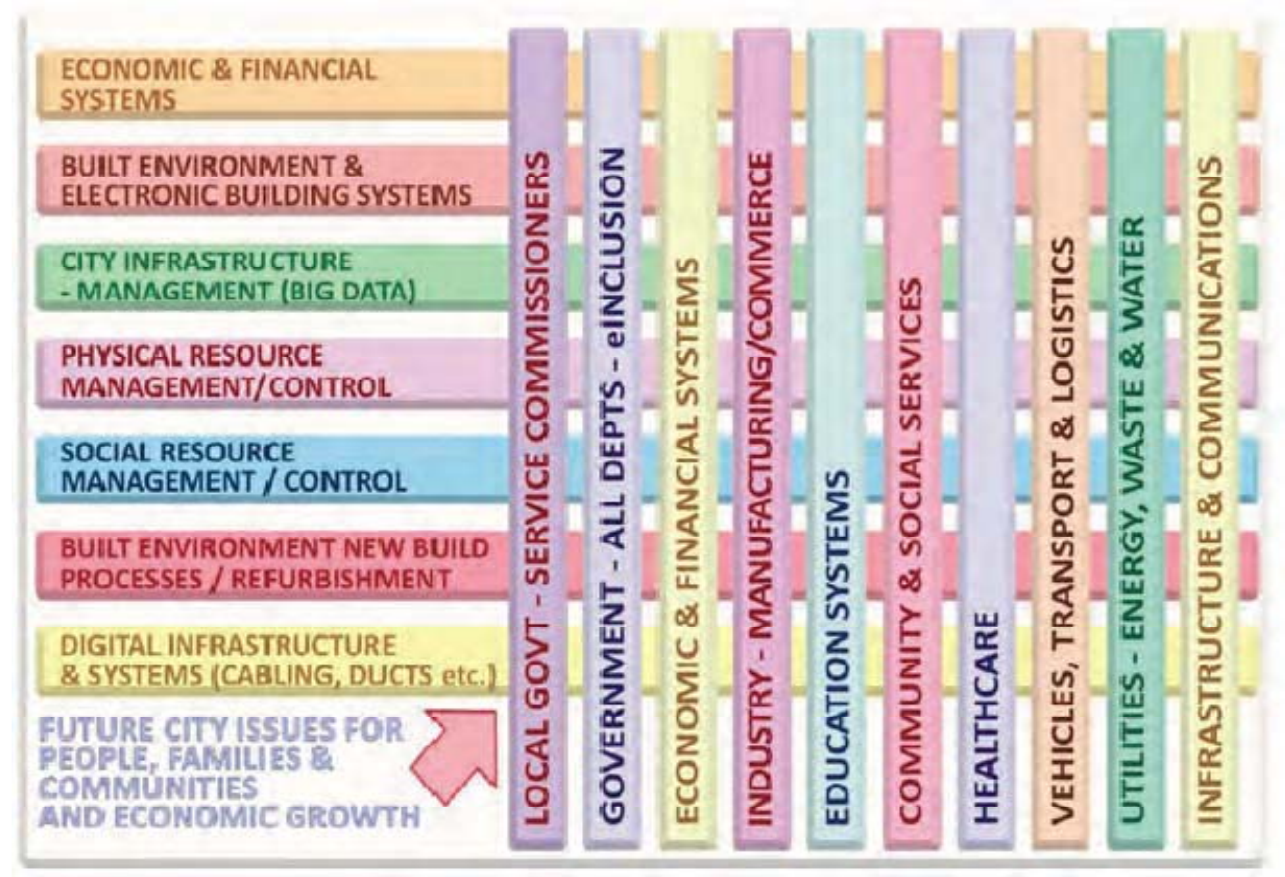

Fig. 3 An enterprise architecture view of smart city.

“silos" . There we see the Education systems in a Smart City context (Fig. 3).

\subsection{Technology Trends}

The future Internet domain landscape comprises a great diversity of technology related topics involved in the implementation of Smart Cities and smart learning environments developments.

Ubiquitous computing is a concept in software engineering and computer science where computing is made to appear everywhere and anywhere. In contrast to desktop computing, ubiquitous computing can occur using any device, in any location, and in any format. A user interacts with the computer, which can 
exist in many different forms, including laptop computers, tablets and terminals in everyday objects.

Networking technologies that are about bringing higher broadband capacity with FTTH, 4G LTE and IMS (IP multimedia systems) provide the infrastructure of the Smart Cities to make all the devices, computers and people can have convenient, reliable, secretive communication paths with each other. Ubiquitous computing is also described as pervasive computing, ambient intelligence, or “everyware".

Open Data in the context of Smart Cities generally refers to a public policy that requires public sector agencies and their contractors to release key sets of government data to the public for any use, or re-use, in an easily accessible manner. In many cases, this policy encourages this data to be freely available and distributable.

Big data is a blanket term for any collection of data sets so large, complex and rapidly changing that it becomes difficult to process using traditional database management tools or traditional data processing applications. A Smart City, as a "system of systems", can potentially generate vast amounts of data. The challenges include capture, curation, storage, search, sharing, transfer, analysis and visualisation.

A GIS (geographic information system) in Smart Cities is used to provide location based services. The implementation of a GIS in Smart City is often driven by city jurisdictional, purpose, or application requirements. GIS and location intelligence applications can be the foundation for many location-enabled services that rely on analysis, visualization and dissemination of results for collaborative decision making.

Cloud computing (public, private or hybrid) is the delivery of computing as a service rather than a product, whereby hared resources, software, and information are provided to computers and other devices as a utility over the Internet.

SOA (service-oriented architecture) is a software design and software architecture design pattern based on distinct pieces of software providing application functionality as services to other applications. SOA can leverage a world of multiple vendors that build systems, which create interoperability and use each other's capabilities.

The E-Government essentially refers to the utilisation of IT, ICTs, and other web-based telecommunication technologies to improve and/or enhance on the efficiency and effectiveness of service delivery.

Embedded networks of sensors and devices into the physical space of cities are expected advancing further the capabilities created by web 2.0 applications, social media and crowd sourcing. A real-time spatial intelligence is emerging having a direct impact on the services cities offer to their citizens. Collective intelligence and social media has been a major driver of spatial intelligence of cities. Social media have offered the technology layer for organising collective intelligence with crowdsourcing platforms, mashups, web-collaboration, and other means of collaborative problem-solving. Smart Cities with instrumentation and interconnection of mobile devices and sensors can collect and analyse data and improve the ability to forecast and manage urban flows, thus push city intelligence forward.

The IoT (internet of things) refers to the interconnection of uniquely identifiable embedded computing like devices within the existing Internet infrastructure. Typically, IoT is expected to offer advanced connectivity of devices, systems, and services that goes beyond M2M (machine-to-machine) communications and covers a variety of protocols, domains, and applications. Internet of Things including sensor networks and RFID is an important emerging strand. These technologies overcome the fragmented market and island solutions of Smart Cities applications and provide generic solutions to all cities. A new round of applications, such as location aware applications, speech recognition, Internet micro 
payment systems, and mobile application stores, which are close to mainstream market adoption, may offer a wide range of services on embedded system into the physical space of cities. Augmented reality is also a hot topic in the sphere mobile devices and smart phones, enabling a next generation location-aware applications and services.

\section{Conceptualising the Emerging Field of Smart Learning Environments}

Given the power and potential of new and emerging technologies, it is time to conceptualise how learning environments can be made smarter (i.e., more effective, efficient and engaging) on a large and sustainable scale. The purpose of this section is to overview a foundational framework for the planning and implementation of smart learning environments, behind which is the convergence of advances and developments in epistemology, psychology, and technology. A few definitions are needed to motivate the discussion [2-4]. Recently smart learning is being defined and studied in diverse ways.

\subsection{Smart Learning Environment Definitions}

The International Association for Smart Learning Environments embraces a broad interpretation of what constitutes a smart learning environment. A learning environment can be considered smart when it makes use of adaptive technologies or when it is designed to include innovative features and capabilities that improve understanding and performance. In a general sense, a smart learning environment is one that is effective, efficient and engaging. According to Spector[5]what is likely to make a learning environment effective, efficient and engaging for a wide variety of learners is one that can adapt to the learner and personalise instruction and learning support. This suggests that appropriate adaptation is a hallmark of smart behavior. The adjective 'smart' isused in everyday language to refer to an action or decision that involved careful planning, cleverness, innovation, and/or a desirable outcome[6].Learning generally involves a stable and persisting change in what a person or group of people know and can do. Intentional learning can occur in a formal context as well as in informal contexts. The notion of an environment suggests a place or surroundings in which something occurs. Whether physical or virtual, an environment can be conducive to or inhibitive of learning. A smart learning environment, in keeping with the emphasis on efficacy, is one that is generally conducive to and supportive of learning.It is emphasised that smart learning is different from e-learning using smart devices[7], and smart learning is defined as "smart device-based intelligent, customised learning service"[8].Broadly defined, smart learning environments represent a new wave of educational systems, involving an effective and efficient interplay of pedagogy, technology and their fusion towards the betterment of learning processes. Various components of this interplay include but are not limited to: (1) Pedagogy/didactics: learning design, learning paradigms, teaching paradigms, environmental factors, assessment paradigms, social factors, policy; (2) Technology: emerging technologies, innovative uses of mature technologies, interactions, adoption, usability, standards, and emerging/new technological paradigms (open educational resources, learning analytics, cloud computing, smart classrooms, etc.); (3) Fusion of pedagogy/didactics and technology: transformation of curriculum, transformation of teaching behaviour, transformation of learning, transformation of administration, transformation of schooling, best practices of infusion, piloting of new ideas. A learning environment can be considered smart when the learner is supported through the use of adaptive and innovative technologies from childhood all the way through formal education, and continued during work and adult life where non-formal and informal learning approaches become primary means for learning. Smart learning environments are neither pure technology-based systems nor a particular pedagogical 
approach. They encompass various contexts, in which students (and perhaps teachers) move from one context to another. So, they are perhaps overarching concept for future academia. This perspective has the potential to overcome some of the traditions of institution based instruction towards lifelong learning.

\subsection{Considerations of Smart Learning Environments Development}

There are several major features of the development of smart learning environments that separates smart learning environments from other advances in learning technologies. These are full context awareness, stacking vs. replacing the LMS, big data and learning analytics, and autonomous decision making.

Full context awareness. Boulanger et al.[9] indicated that smart learning environments involve context awareness that can combine a physical classroom with many virtual learning environments. This could provide full context awareness by combining smart learning environments with holistic Internet of Things and ubiquitous sensing devices, e.g., wearable technologies such as smart watches, brainwave detection, and emotion recognition [10]. Full context awareness enables smart learning environments to provide learners with authentic learning contexts and seamless learning experiences to fuse a variety of features in the e-learning environments. The system includes learning management systems, mobile and ubiquitous learning systems, various artificial intelligence based adaptive and intelligent tutoring/learning systems. These systems would assist teachers and instructors in direct monitoring of the learning environment, understand learners' conditions and give learners real-time adaptive assistance, while at the same time facilitating independent learning for the learners [11].

Stacking vs. Replacing the LMS. While many organizations have grown beyond the current capacities of their Learning Management Systems, there are significantly fewer organizations choosing to make the major capital and implementation investment of replacing their entire enterprise learning technology. Rather, we are seeing more "Stacking" which means accepting the role of the existing LMS as the base system for the organization and then adding Stacks or Layers on top that will create added and more targeted functionality. Some of the Learning Stacks include: Competency or Talent Management Layers; Assessment or Feedback Layers; Compliance or Regulatory Layers; Career Development Layers; Collaboration and Social Networking Layers, Gamification or Engagement Layers, Globalisation Layers. In other words, some organizations are shifting from replacing their LMS to adding these technologies on top of the LMS. It might be called a "LMS Inside" approach as extensions of the LMS, using the core code for transaction tracking and shared data exchange - but the functionality is found in the layer.

Big data and learning analytics. Smart learning environments need to consider advanced data manipulation techniques such as employing big data and learning analytics to collect, combine and analyse individual learning profiles in order to scientifically generalise and infer each individual learning need in real time in ubiquitous settings that encompass both physical and online activities. Learning analytics by using big data can monitor individual learners' progress and behaviour continuously in order to explore factors that may influence learning efficiency and effectiveness [12, 13].

Autonomous Decision Making and Dynamic Adaptive Learning. Another important feature of smart learning environments, which is different from other learning environments, is their autonomous knowledge management capability that enables them to automatically collect individual learners' life learning profiles. As Kay mentioned [14], smart learning environments can precisely and autonomously analyse learner's learning behaviours in order to decide in real time, for example, what interactions with the physical 
environment to recommend to the individual learners to undertake various learning activities, the best location for those activities, which problems the learners should solve at any given moment, which online and physical learning objects are the most appropriate, which tasks are the best aligned with the individual learner's cognitive and meta-cognitive abilities, and what group composition will be the most effective for each group member's learning process. Such autonomous decision-making and dynamic adaptivity has the potential to generalise and infer learners' learning needs in order to provide them with suitable learning conditions. It is a challenge for smart learning environments to collect these data about the learners and their environment from disparate sources in both physical and online components of the ubiquitous settings.

\subsection{Smart Learning Environments Foundation Areas}

According to Spector[5] social constructivism, psychology and technology are the foundation areas that provide meaningful and convergent input for the design, development and deployment of smart learning environments. At a high level, social constructivism provides a coherent approach to many human activities, including learning design and technology research and practice. It consists of two primary tenets that describe how humans develop knowledge and expertise. The first tenet involves the creation of mental models when encountering new or unusual or otherwise unexplained experiences. Simply stated, this is the notion that people create internal representations to make sense of their experience. This perspective puts the individual at the centre of knowledge and skill development, and it implies that individuals may develop knowledge and skills differently. The second tenet from a philosophical perspective involves the role of language as a critical mediator in learning and knowledge development. The underlying idea is that interaction with others, especially in the form of discourse, contributes to how knowledge is developed.
Taken together, these two tenets provide a general description of how people come to know and understand their worlds - namely by a process of creating internal mental representations and then sharing ideas formed on the basis of those representations with others through appropriate languages and media. Because social constructivism provides a coherent philosophical foundation for learning and instruction, it should be recognised as a pillar of any smart learning environment.

The role of psychology in learning and instruction is well recognised. The two main streams of educational psychology have been behaviourism and cognitivism. Behaviourism emphasises things that can be observed and measured as a way to understand and predict human behaviour. The emphasis on outcomes is a valuable contribution of behaviourism that is worth retaining in understanding smart learning environments. Cognitivism emphasises the need to understand mental processes that underlie and can explain many human behaviours. Social psychology emphasises the effects and impact of others on how people think and behave. There is a strong parallel between social psychology and its relationship to behavioural and cognitive psychology. People do not live and learn in isolation from others. A smart learning environment will take this fact into account explicitly and in meaningful ways.

Learning technology regularly undergoes changes in response to changes in mainstream models of human cognition and learning. Modern information and communications technologies are expected to support and strengthen the processes of creating, transforming and sharing knowledge in a smart environment. This often requires an innovative use of a technology in an engaging and flexible manner.Web 2.0 and Social 3.0 have captured the interest and the imagination of students, educators and researchers.

Web 2.0 presents opportunities for teachers to build higher levels of engagement in the classroom. Giving students the ability to think critically about transferring 
skills and knowledge to new creations teachers use Web 2.0 to encourage students to view themselves as active agents in the transfer process. The impact of Web 2.0 has instrumentally changed the way students learn and in return the way teachers must teach. The number of Web 2.0 tools continues to grow while utilisation of these tools supports constructivist pedagogy. The interactive nature of these technologies lends itself well to collaborative learning, which motivates students, creates a "safer" learning environment, and enhances knowledge and skills. Users become creators, collaborators and actively engaged with Web 2.0 tools.

As broadband penetration increases, people become more empowered to connect with each other on their own terms. "Social Media" as we know is just to describe the nature of sharing online. From Facebook and Twitter to Snapchat and Whatsapp, the apps for online sharing vary as much as the Web 2.0 products. While there is power in collaboration, concern exists too. The impact of these technologies upon culture, education, and knowledge is clear. According to Norris and Soloway [15] Social 3.0 is defined as: two or more individuals verbally conversing; while those two or more individuals are engaged doing "something" inside an app or in a Web page; and while those two or more individuals are either co-located, or more interestingly, not co-located. Thinking of tools in terms of students' level of knowledge creation, the hierarchy of revised Bloom's Taxonomy (Fig. 4) will enhance appropriate understanding.

\section{Smart Learning Design Model}

There is no single or simple way to characterise knowledge development. People create internal representations and then talk about those representations with others along various paths to understanding. People are smart in different ways, at different times, and in different circumstances. In response to the uncertainty regarding opportunities and challenges facing education systems in transforming the classroom into effective teaching and learning environments, we have explored innovative uses of technology that supports new ways to explore, learn, and share knowledge in technology enabled smart learning environments. Transforming the process

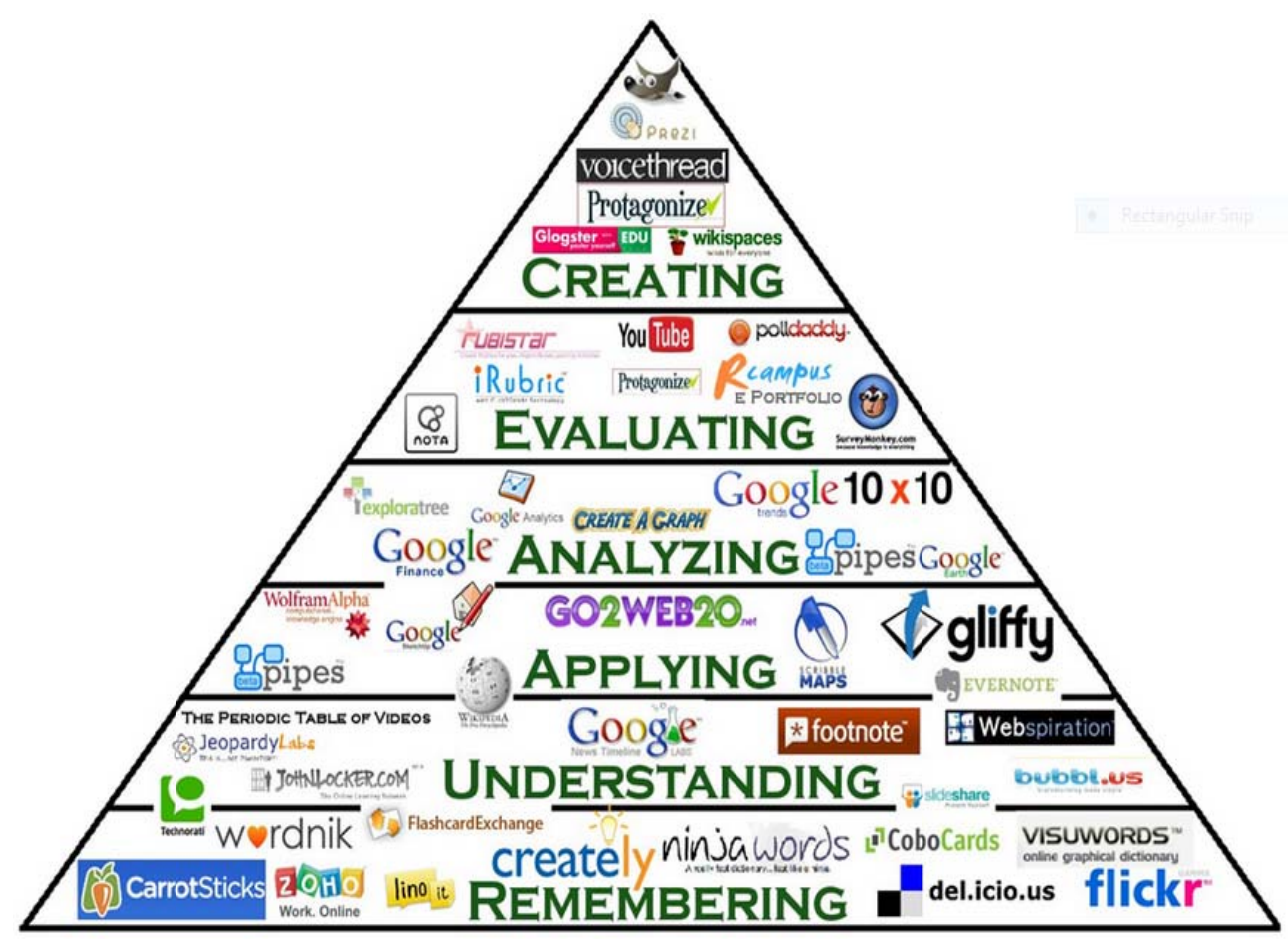

Fig. 4 The hierarchy of revised Bloom's Taxonomy supported by technologies. 
of teaching and learning means that teachers create fundamentally different learning environments and promote interactivity, socialisation; externalisation, combination and internalisation thus create knowledge as stated by Nonaka and Takeuchi [16]. On this point of view learning design and developing of interactive learning scenarios/activities are critical for the successful development of any learning environment supported by today's physical environments that are enriched with digital, context-aware and adaptive devices, to promote better and faster learning (Fig. 5).

The interplay between dynamic knowledge conversion processes in technology enabled smart learning environments enhanced by innovative learning scenario is the basis behind the proposed model, which is depicted in Fig. 6. The model reflects an emphasis on the knowledge creation that can foster learning outcome. Drawing on IMS LD specifications [17] and current technology innovation, we assumed that the commitment and the creativities of individual users (teachers) are crucial to developing new practices and approaches. The scenarios can be defined as narrative descriptions of preferable learning contexts that take into account user stories, including the description of resources and the functionalities needed, the interactions they have, the tasks they perform and the aims of their activities. The SECI model is the essence of knowledge management. With this mode, we can grow to complex model creating complementary models and using support tools. In each

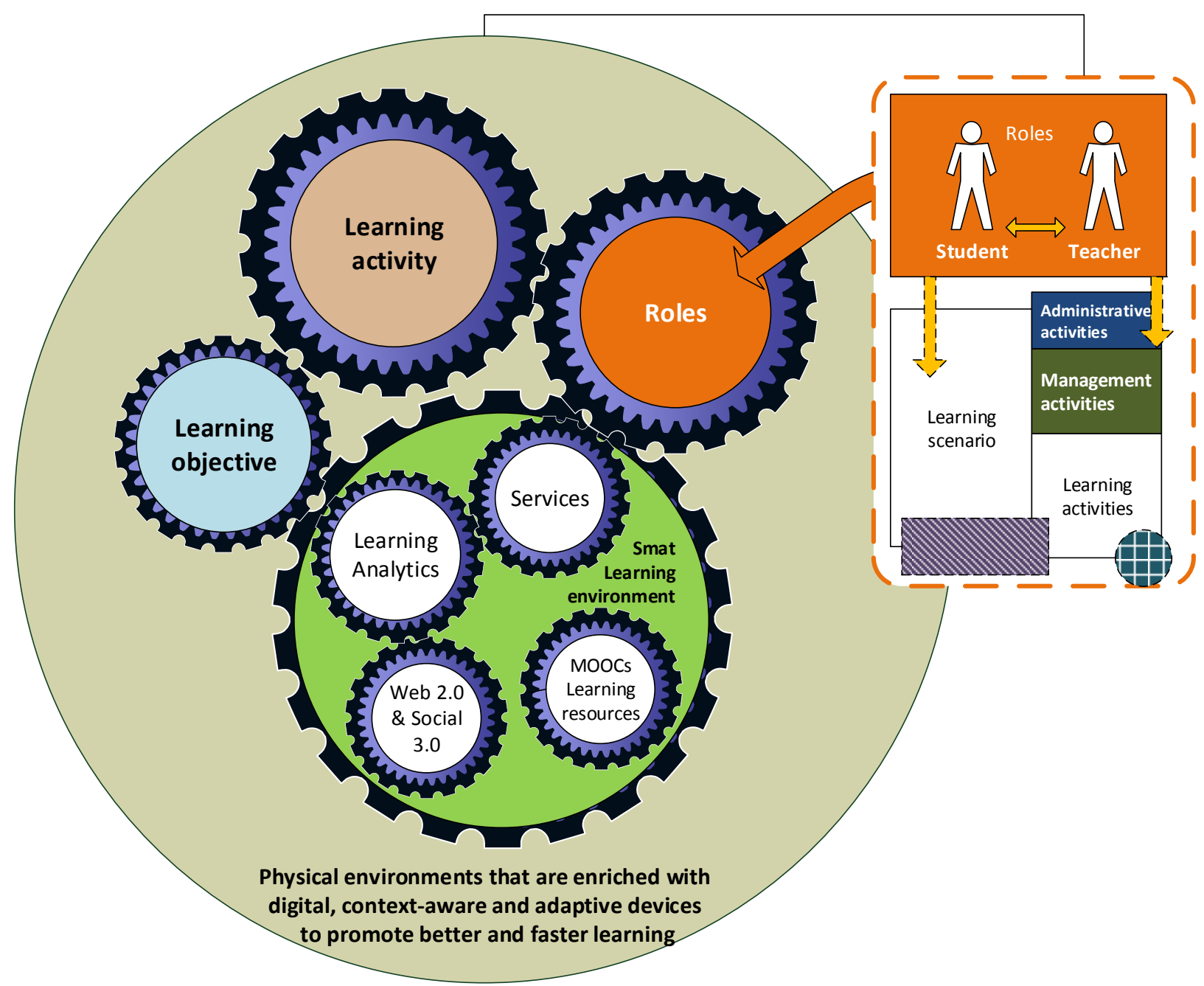

Fig. 5 Innovative learning scenario/activities supported by smart learning environments. 


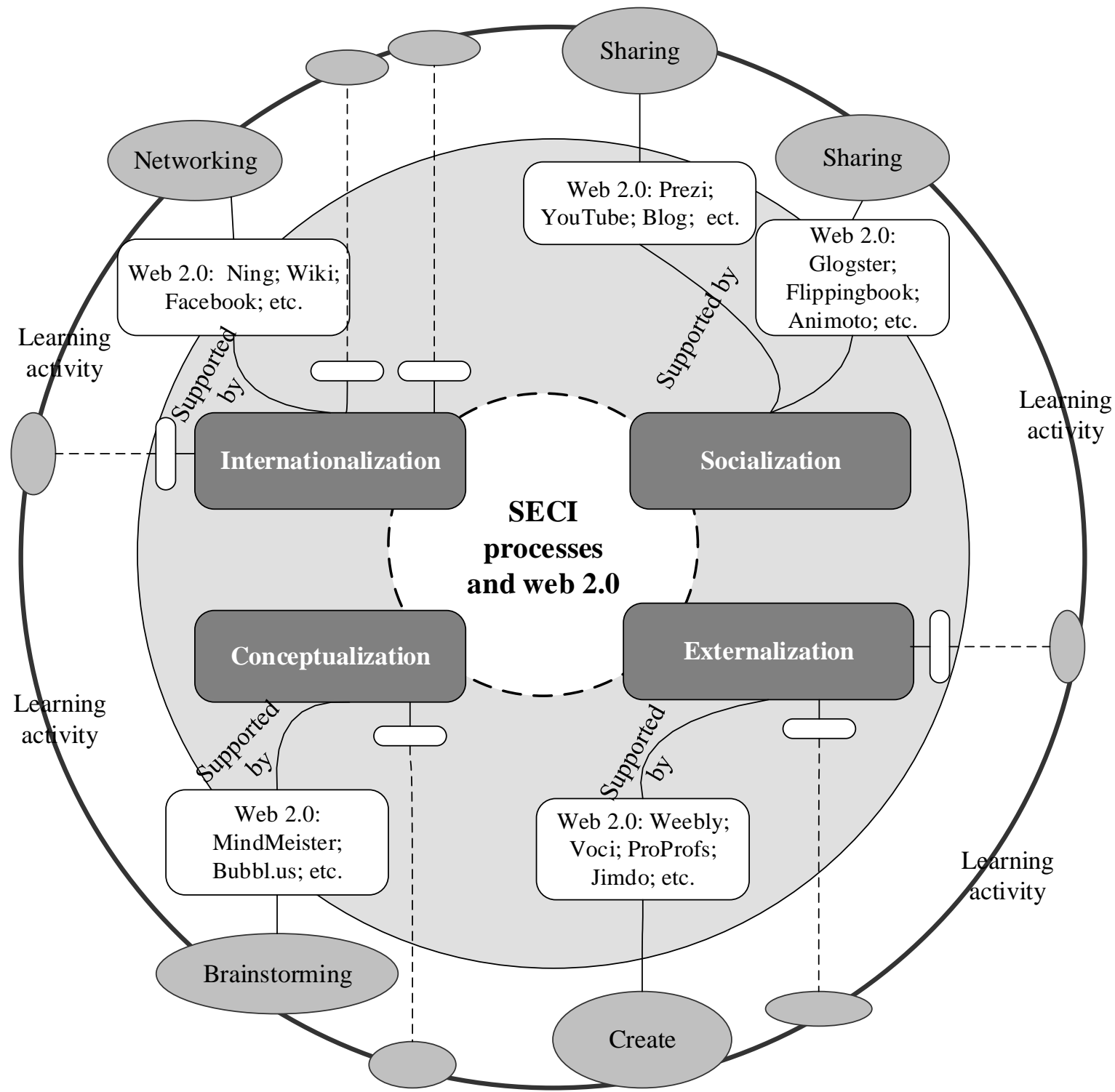

Fig. 6 SECI 2.0 -Dynamic knowledge conversion processes in technology enabled smart learning environments.

of the four SECI knowledge conversion stages diversity learning activities supported by smart technology can take place. The central thought of the model is that knowledge held by individuals is shared with other individuals so it interconnects to a new knowledge. The spiral of knowledge or the amount of knowledge grows all the time when more rounds are done in the model. Knowledge creation can be viewed as a bottom-up spiral process, starting with the sharing of tacit knowledge at the individual level and moving to crystallisation of the knowledge at the group level and then on to the organisational level. Then the combination process deductively produces increased collective understanding, which is then internalised by reflection and embodied into increased individual understanding. In each one SECI stages a wide range of smart learning scenarios and activities performed in collaborative and knowledge platforms (Animoto, Proshow, Prezi, Vuvox, Screenr, Quiz-Creator, Bitrix24, Flipsnack, ProProfs, Voci...) and supported by smart technologies and services can be applied.

Smart technology attuned to the emergent nature of thinking and learning not just points to greater control over the students, but emphasis shifts to control of 
productive interaction between students, teachers, ideas and technologies. The rapid progress of mobile, wireless communication and sensing technologies has enabled the development of context-aware ubiquitous learning environments, which are able to detect the real-world learning status of students as well as the environmental contexts.

\section{Case Study}

\subsection{Synergy with the FP7 FORGE Project "Forging Online Education through FIRE"}

The University has started to adopt co-op and internship programs with FP7 FORGE project "Forging Online Education through FIRE" (http://ict-forge.eu/) to facilitate experiences, particularly in computer science programs. The EU FIRE (future internet research and experimentation) initiative creates an open research environment that facilitates strategic research and development of new Future Internet concepts, giving researchers the tools they need to conduct large-scale experiments on new paradigms. The FORGE project introduces the FIRE experimental facilities to the eLearning community, in order to promote experimentally driven research in education by using experiments as an interactive learning and training channel both for students and professionals. FORGE provides learners and educators with access to world-class experimentation facilities and high quality learning materials via a rigorous production process. In FORGE, we focus on development methodologies and best practices for remote experimentation performed on top of FIRE facilities.

The University delivers the UXD (user experience design) for IoT Specialisation in the Software Engineering Master Program, which heavily relays on present research and the FORGE eLearning methodology and tools having the opportunity to study in depth various aspects of networking protocols and infrastructure, watch instructional movies and screen casts, as well as conduct experiments using the
FIRE infrastructure. The goal of the Specialisation program is to provide graduates with theoretical knowledge, practical skills and tools necessary to begin the professional practice of designing user-centric next generation devices. Graduates of this program will be able to implement a holistic, multidisciplinary approach to the design of user interfaces for Internet of Things products, defining their form, behaviour, and content. This Specialisation covers the foundation of UXD (Emotive UXD, Personalized UXD, and Visual UXD) and the development of Internet of Things products and services-including devices for sensing, actuation, processing, and communication-to help them develop skills and experiences they can employ in designing novel systems. The Specialisation has theory and lab sections. In the IoT lab sections students will learn hands-on IoT concepts such as sensing, actuation and communication. The FORGE model and methodology employed for the development of interactive lab in the field of Internet of Things aimed at fostering remote experimentation with real production system installed in the Smart City, such as Smart Santander. A FORGE enabled course will keep a low complexity for setting up simple experiments on FIRE facilities, for both learners and teachers, while making them aware that they are using real resources remotely. On one hand, teachers will use FORGE tools to create simple experimentation scenarios and inject them into their courses. These tools will ease the process of browsing, reserving and scheduling FIRE resources while pointing and using tools that FIRE already offers. On the other hand learners will be initially guided to focus on studying a specific subject and then, later on, experiment with aspects of this subject onto a real infrastructure. At the end of the course, learners will be guided to re-create the experimentation environment by using the FIRE facility. Thus, learners in the end will have a good understanding of what is FIRE, what it offers and how it can be used. To promote the concept of experimentally 


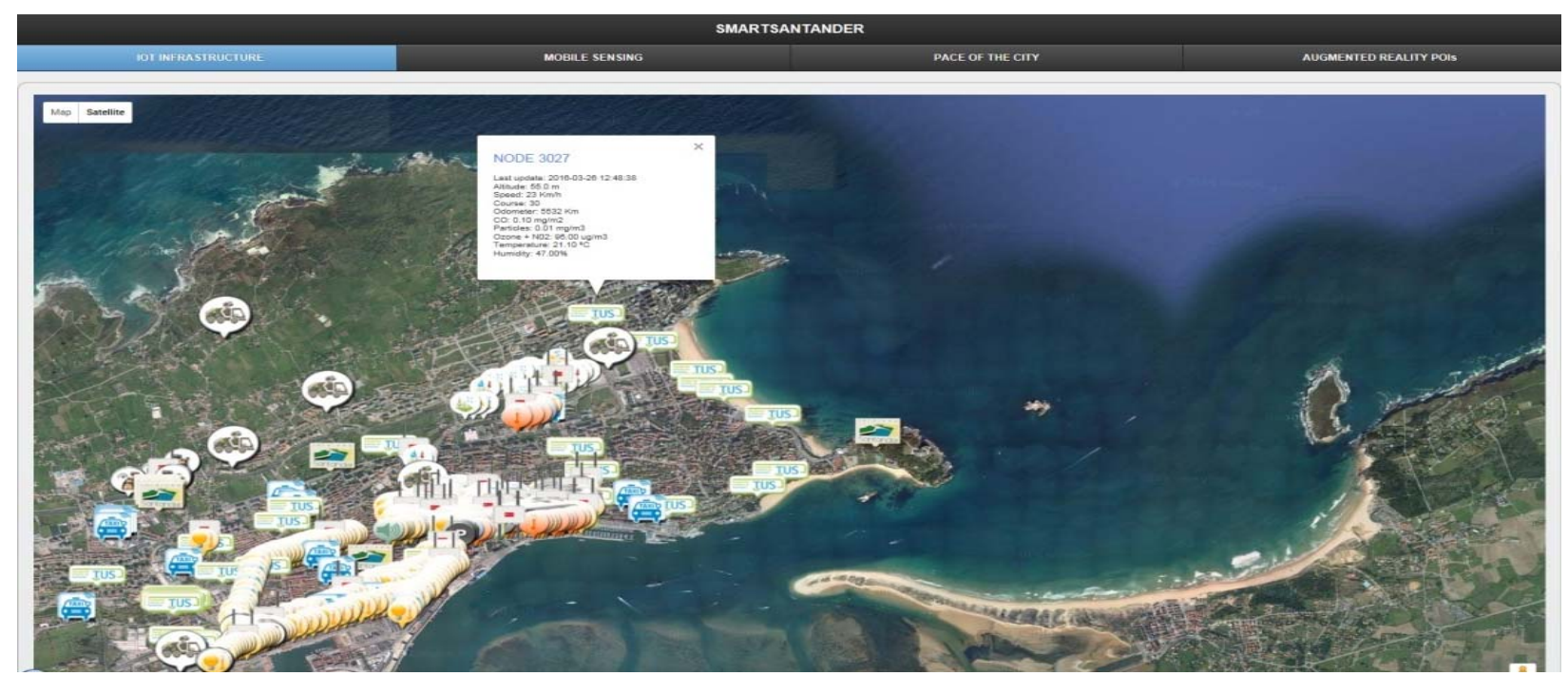

Fig. 7 Smart Santander IoT infrastructure.

driven IoT research in education following requirements have to be considered: Realism of experimentation environment; Heterogeneity of IoT devices; Adequate scale; Mobility support from controlled to realistic; Concurrency; Repeatability and replayability; Real end user involvement in the experimentation cycle; Federation with other Internet research facilities.

As an illustration of experimental learning on IoT, a learning scenario entitled "Monitoring the environmental parameters in Smart City” is presented, which proofs the power of FORGE methodology and infrastructure for building remote labs and delivering them to students.

\subsection{Learning Scenario: Monitoring the Environmental Parameters in Smart City}

Aim: In this experimental lab students will learn hands-on IoT concepts, such as sensing and communication in Smart City.

Smart environment: Smart Santander infrastructure and its interactive online site, which is conceived as a 3-tiered approach and defined next:

(1) IoT node: Responsible for sensing the corresponding parameter (temperature, CO, noise, light, car presence, soil temperature, soil humidity). The majority of them are integrated in the repeaters, whilst the others standalone communicating wirelessly with the corresponding repeaters (it is the case for the parking sensor buried under the asphalt). For these devices, due to the impossibility of powering them with electricity, they must be fed with batteries.

2. Repeaters: These nodes are high-rise placed in street lights, semaphores, information panels, etc., in order to behave as forwarding nodes to transmit all the information associated to the different measured parameters. The communication between repeaters and IoT nodes performs through 802.15.4 protocol.

3. Gateways: Both IoT nodes and repeaters, are configured to send all the information (through 802.15.4 protocol), experiment-driven as well as service provision and network management to the gateway. Once information is received by this node, it can either store it in a database which can be placed in a web server to be directly accessed from internet, or send it to another machine (central server), through the different interfaces provided by it (WiFi, GPRS/UMTS or ethernet).

Within the Smart Santander project more than 2,000 environmental monitoring sensors have been already deployed. These sensors are monitoring CO index, temperature, noise level and light intensity.

Learning activities:

(1) Navigate through the various parts of the Smart 
Santander (http://maps.smartsantander.eu/) and become familiar with the capabilities of the freely accessible platform tags - IoT infrastructure, Mobile Sensing, Pace of the City, Augmented Reality POIs and play with consideration of various parameters.

(2) Explore the Smart Santander IoT infrastructure and examine the set of parameters of the environment, the system is able to monitor.

(3) Find on the Internet intelligent sensors or complete devices that can measure the same set of parameters.

(4) Explore the features of smart sensors for air pollution, for example $\mathrm{C} 02$, O3, particulate matter and $\mathrm{ZO} 2$.

(5) Design of a "Network of sensors and system for continuous monitoring of the air".

Further, to support the learning process a hybrid cloud infrastructure has been established, which integrates variety of collaboration platforms and eLearning systems. The cloud based infrastructure enables innovative learning scenario execution and monitoring.

\section{Conclusion}

This paper discusses a vision and some steps toward development of smart learning environments. These environments are expected to break the boundaries of the traditional learning and enable the detection of the learner's location, environment, proximity and situation. This would provide a fully contextualised learning process in order to provide learners with learning scenarios in their own living and work environments, leading to significantly better learning experiences.

\section{References}

[1] ISO/ IEC JTC1, 2014. Smart Cities Preliminary Report,www.iso.org.

[2] Richey, R. C., Klein, J. D., and Tracey, M. W. 2011. The Instructional Design Knowledge Base: Theory, Research, and Practice. NewYork: Routledge.

[3] Seel, N. M. etc.2012.The Encyclopedia of the Sciences of Learning. New York: Springer.
[4] Spector, J. M.2012. Foundations of Educational Technology: Integrative Approaches and Interdisciplinary Perspectives. New York: Routledge.

[5] Spector, J. M. 2014. "Conceptualizing the Emerging Field of Smart Learning Environments.”Smart Learning Environments 1 (2).

[6] Spector, J. M. etc.2015. Encyclopedia of Educational Technology. Thousand Oaks: Sage.

[7] Lin, Y.-T., Huang, Y.-M., Kinshuk, Q.-T: Location-Based and Knowledge-Oriented Microblogging for Mobile Learning. Framework, Architecture, and System, pp. 146-150 WMUTE (2010)

[8] Korea Education and Research Information Service: Issues Analysis of the Learning Management System in Smart Education, RM 2012, vol. 18, pp. 11(2012), ITPLUS.: http://www.ktoa.or.kr/.

[9] Boulanger, D. etc. 2015. "Smart Learning analytics.”In Emerging issues in smart Learning, edited by G. Chen, V. Kumar, Kinshuk, R. Huang, \& S. C. Kong (Eds.), Berlin Heidelberg: $\quad$ Springer. Retrieved from http://link.springer.com/chapter/10.1007/978-3-662-4418 8-6_39.

[10] Li, B. P., Kong, S. C., and Chen, G.2015. “A Study on the Development of the Smart Classroom Scale.” In Emerging Issues in Smart Learning. Heidelberg: Springer. Retrieved from http://link.springer.com/chapter/10.1007/978-3-662-4418 8-6_6.

[11] Hwang, G. J. 2014. "Definition, Framework and Research Issues of Smart Learning Environments - a Context Aware Ubiquitous Learning Perspective.”Smart Learning Environments 1 (1): $4 . \quad$ doi: 10.1186/s40561-014-0004-5.

[12] Kumar, V. S., Kinshuk, D., Clemens, C., and Harris, S. 2015. "Causal Models and Big Data Learning Analytics.”In Ubiquitous Learning Environments and Technologies, Heidelberg: Springer.

[13] Kumar, V. S. etc. 2015. "Big Data Learning Analytics: A New Perpsective.” In Ubiquitous Learning Environments and Technologies, Heidelberg, Germany: Springer.

[14] Kay, J. 2008. "Life-long Learning, Learner Models and Augmented Cognition.”In Intelligent Tutoring Systems, edited by Woolf, B. P., Aimeur, E., Nkambou, R., and Lajoie, S. (Eds.), Berlin: Springer.

[15] Norris, C., and Soloway, E. 2014. "Web 2.0 to Social 3.0: The Next Big Thing.”The Journal

[16] Nonaka, I. and Takeuchi, H.1995.The Knowledge-creating Company: How Japanese Companies Create the Dynamics of Innovation. New York Oxford: University Press.

[17] IMS Learning Design Specification http://www.imsglobal.org/learningdesign/index.htm. 